\title{
NEW CONCEPTS AND TEST METHODS OF CURVE PROFILE AREA DENSITY IN SURFACE: ESTIMATION OF AREAL DENSITY ON CURVED SPATIAL SURFACE
}

\author{
HONG SHEN \\ Department of Pathology, The First Military Medical University, Guangzhou, 510515, P. R. China \\ e-mail: hongshen@fimmu.edu.cn \\ (Accepted February 21, 2001)
}

\begin{abstract}
The concepts of curve profile, curve intercept, curve intercept density, curve profile area density, intersection density in containing intersection (or intersection density relied on intersection reference), curve profile intersection density in surface (or curve intercept intersection density relied on intersection of containing curve), and curve profile area density in surface $\left(A_{S}\right)$ were defined. $A_{S}$ expressed the amount of curve profile area of $Y$ phase in the unit containing surface area, $S(X)$. The test methods and formulas were deduced, e.g.: $A_{S}=I_{C I} / I_{C}=L_{C I} / B_{C}$. In the formula, $I_{C I}$ and $I_{C}$ indicated the intersection numbers of test line with curve intercepts (or profiles in 2D) and with the containing curve respectively. $B_{C}$ denoted the length of containing curve, $L_{C I}$ being the length of curve intercepts. The formula means that $A_{S}$ are equal to the curve intercept density and equal to the curve profile intersection density in surface. Issues about $A_{S}$ application and its correction for shrinking tissues were discussed. Example for testing $A_{S}$ was given.
\end{abstract}

Keywords: curve profile area density in surface, curve intercept density, stereology, intersection density relied on intersection reference.

\section{INTRODUCTION}

As we know that area is a basic parameter important to describe quantitatively a structure. The morphometrical parameters for describing area-related feature include area, area density, surface area, surface density and the ratio of surface to volume. Area $(A)$ is used to describe the absolute size of plane structure. Area density $\left(A_{A}\right)$ denotes the amount of area of interested phase in unit reference area. Surface area $(S)$ is a three dimensional index to describe the absolute surface size of interested phase. Surface density $\left(S_{V}\right)$ means the amount of surface area of interested phase in unit containing space volume. The ratio of surface to volume, $S(X) / V(X)$, denote the ratio of surface area of $X$ phase (or particles) to the volume of the same $X$ phase (or particles) itself (Weibel, 1979; 1980; Collan, 1984; Shen and Shen, 1991; Howard and Reed, 1998). In practice, some biostructures often distribute and take up certain area on or in its containing surface structure, such as desmosomes in cells membrane, nuclear pores in nuclear membrane etc. It is clear that aforesaid area-related parameters can not describe the amount of surface area occupied by particles structure which distribute on or in surface area of membrane or membrane like structure in unit surface area of correspondent membrane or membrane like structure, for example, the amount of basement membrane surface area occupied by some kind of glandular epithelium cells in unit basement membrane surface area of the gland, the amount of cell membrane surface area occupied by desmosomes in unit surface area of cell membrane, the amount of reticulum surface area occupied by ribosomes in unit surface area of rough endoplasmic reticulum. This kind of problems as aforesaid are generalized in this paper as the problem of seeking curve profile area density in surface, i.e.: $S(Y) / S(X)$. Then, how to solve this kind of problem?

\section{DEFINITION AND FORMULA DEDUCTION}

Let $A$ for planar area, $S$ for spatial surface area, $A_{A}$ for areal density, $S(X) / V(X)$ for the ratio of surface to volume of a set of particles, $S_{V}$ for surface density, $S(Y) / S(X)$ for the surface density of a spatial curved surface $(Y)$ embedded in a spatial curved surface $(X)$, $S(Y \cap T)$ for profiles of the spatial curved surface $Y(T$ being a probe), $S(Y)=S(Z \cap X)$ for the surface of the intersection of two set $(Z, X)$ (Weibel, 1980). 
Definition 1: A spatial curved surface $X$ containing interesting structures in its surface is defined in the paper as containing spatial curved surface or containing surface (or containing curve when in 2D). Let $X$ be a curved spatial surface 3D set containing or intersecting a subset $Y$ of interesting spatial curved structures, the boundary of $X$ (or $\delta X$ ) will be called a containing surface (or a containing curve in case $X$ being a 2D set).

Definition 2: The curved intersection formed by a containing surface intersecting with 3D structure (Fig. 1(3),(4) is defined in the paper as curve profile (Fig.1 (5), (6)). Let $Z$ be a 3D structure and $X$ a containing surface, then the curved intersection:

$$
Y=Z \cap \delta X
$$

is called a curve profile. Based on the features of $Z$, volumic or unvolumic (or shell), which intersect with the containing surface, the curve profiles can be divided into two kinds: the solid curve profile (Fig1(5) and the empty curve profile (Fig. 1(6) whether $Z$ is a volumic or unvolumic (or shell) structures. The curve profiles formed by desmosomes or hemidesmosomes intersecting with cell membrane are just a solid curve profiles. And the nucleopores are empty curve profiles.

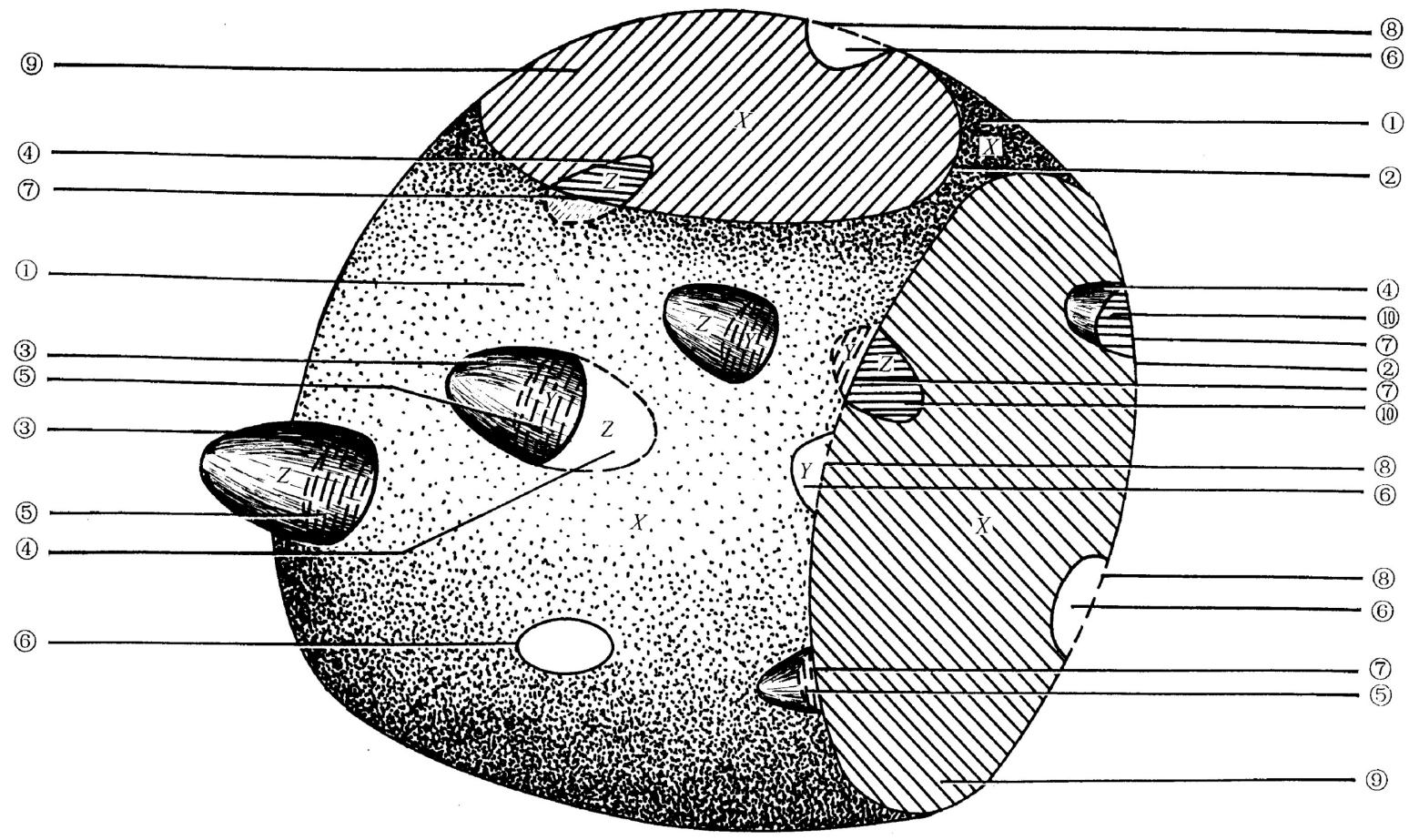

Fig. 1. Show containing surface of $X(1)$, containing curve lines ( (2) formed by containing surface intersecting with cross sections), particles $Z$ ( (3) for particles towards outside of the containing surface, (4) for particles towards inside of the containing surface), curve profiles $Y$ ( (5) for solid curve profiles formed by curve surface intersecting with solid particles which are towards outside or/and inside of the curve structure or just crossing the curve surface, (6) for empty curve profiles formed by breaches or holes in containing surface), curve intercepts (จ for solid curve intercepts (black line) formed by a curve line intersecting with solid curve profile, (8) for empty curve intercepts (dotted lines) formed by a curve line intersecting with empty curve profiles), cross section in $2 D$ plane ( (Q) for the cross section of containing surface structure X and (10 for the cross section of $3 D$ particles Z.). 
Definition 3: The intercept formed by a curve profile intersecting with a 2D plane was defined as curve intercept (Fig. 1(7), (8)). Check the distribution of section as IUR, and let $T_{2}$ be a planar probe (DeHoff RT, 2000), then the intersection

$$
Y \cap T_{2}=[Z \cap \delta X] \cap T_{2}
$$

will be called a curve intercept (empty or solid). If a solid curve profile intersecting with a $2 \mathrm{D}$ plane, the corresponding curve intercept is named as solid curve intercept (Fig. 18), and if an empty curve profile intersecting with a 2D plane, the corresponding curve intercept, which is invisible in fact, is named as empty curve intercept (Fig. 18).

Definition 4: The length of curve intercepts in unit length of containing curve, when $X$ being a 2D set, is defined as the curve intercept density (CID), i.e.:

$$
C I D=\frac{\sum\left\{L\left[Y \cap T_{2}\right]\right\}}{\sum\left\{L\left[\delta X \cap T_{2}\right]\right\}}
$$

In Eq. 3, L denotes the length of curves. The formula can rewrite as:

$$
C I D=\frac{\sum L_{C I}}{\sum B_{C}}
$$

In the formula, $L_{C I}$ is the length of curve intercepts, $B_{C}$ is the boundary length of containing curve, which can be membrane like structure, in 2D section. It is clear that $C I D$ is a parameter with no physical unit.

Definition 5: Let $V(X)$ be the volume of the containing space bounded by a curved spatial surface, then the amount of curve profiles surface area of $Y, S$ $(Y)$ or $C P A$, in unit volume of containing space is defined as the curve profile area density $\left(C P A_{V}\right)$, that is:

$$
C P A_{V}=\frac{S(V)}{V(X)}
$$

The unit of $C P A_{V}$ may be $\mathrm{cm}^{2} / \mathrm{cm}^{3}, \mathrm{~mm}^{2} / \mathrm{mm}^{3}$, $\mu \mathrm{m}^{2} / \mu \mathrm{m}^{3}$, etc.

Definition 6: The amount of intersections of intercepts from subset $Y$ in containing curve intersecting with test line in unit intersections of containing curve intersecting with test line is defined as intersection density in containing intersection, or intersection density relied on intersection reference, represented by $I_{I}$. Check the distribution of test lines in
3D IUR distribution and let $T_{l}$ be an IUR probe line and $I$ denotes the number of intersections. Then:

$$
I_{I}=\frac{\sum\left\{I\left[Y \cap T_{1}\right]\right\}}{\sum\left\{I\left[X \cap T_{1}\right]\right\}}
$$

is intersection density in containing intersection, or intersection density relied on intersection reference. The formula can be rewritten as:

$$
I_{I}=\frac{\sum I_{Y}}{\sum I_{C}}
$$

In the formula, $I_{Y}$ is the amount of intersections of intercepts from subset $Y$ in containing curve intersecting with test line; $I_{C}$ is the amount of intersections of containing curve intersecting with test line.

According to the definition of $I_{I}$, the amount of intersections of curve intercepts (or curve profiles) in containing curve (or surface) intersecting with test line in unit intersections of containing curve (Fig. 1(2), or containing surface of Fig. 1(1) intersecting with test line, is named as curve intercept intersection density relied on intersection of containing curve (containing intersection), or curve profile intersection density in surface, represented by $I_{I S}$ in the paper. That is:

$$
I_{I S}=\frac{\sum I\left\{\left[\left(Y \cap T_{2}\right) \cap T_{1}\right]\right\}}{\sum\left\{I\left[X \cap T_{1}\right]\right\}}
$$

So, $I_{I S}$ can be calculated by:

$$
I_{I S}=\frac{\sum I_{C I}}{\sum I_{C}}
$$

In the formula, $I_{C I}$ is the intersections of curve intercepts (or curve profiles in 2D section) intersecting with test line, $I_{C}$ is the intersections of containing curve intersecting with test line.

Definition 9: The amount of curve profile area $(S(Y)$ or $C P A)$ in unit surface area of containing surface, $S(X)$ or $S_{C}$, is defined as curve profile area density in surface, represented by $A_{S}$. The definition formula is that:

$$
A_{S}=\frac{S(Y)}{S(X)}
$$

Eq. 10 can be rewritten as: 


$$
A_{S}=\frac{C P A}{S_{C}}
$$

It is clear by above formula that $A_{S}$ is a parameter with no physical unit. Because $C P A$ and $S_{C}$ are hardly to measure or get directly from 3D under microscope, the key question is how to test $A_{S}$ based on 2D section? The following deduction is to solve this problem.

Dividing separately numerator and denominator of Eq. 11 by $V_{C}$, containing space volume, then we can get that:

$$
A_{S}=\frac{C P A / V_{C}}{S_{C} / V_{C}}=\frac{C P A_{V}}{S_{V}}
$$

In the formula, $S_{V}$ is the surface density for the containing curve (or containing surface) itself, or $S(X) / V(X)$ for the ratio of surface to volume of a set of $X$, i.e.: the amount of containing surface area in unit containing space volume, or the ratio of surface area of containing curve to the volume of containing

space. Based on the principle and methods of stereology (Weibel, 1979, 1980; Collan, 1984; Shen and Shen, 1991; Howard and Reed, 1998):

$$
S_{V}=\frac{S(\delta X)}{V(X)}=2 \frac{\sum\left\{I\left(X \cap T_{1}\right)\right\}}{\sum\left\{L\left(X \cap T_{1}\right)\right\}}=\frac{4 \sum\left\{B\left(\delta X \cap T_{2}\right)\right\}}{\pi \sum\left\{A\left(X \cap T_{2}\right)\right\}}
$$

or:

$$
S_{V}=\frac{2 \sum I_{C}}{\sum L_{C}}=\frac{4 \sum B_{C}}{\pi \sum A_{C}}
$$

In the formula, $X$ is the containing space and $\delta X$ its boundary; $L_{C}$ is the length of test line in containing space; $I_{C}$ is the numbers of intersections of containing curve (including the part of curve intercepts) intersecting with test line; $A_{C}$ is the area of containing space in 2D plane; $B_{C}$ is the length of boundary or curve line of the containing surface in $2 \mathrm{D}$ slices. Because $C P A$, the curve profile area, is a part of surface area in containing surface, $S_{C}$, therefore the curve profile area density can be calculated similar as surface density, that is:

$$
C P A_{V}=\frac{S(Y)}{V(X)}=2 \frac{\sum\left\{I\left(Y \cap T_{1}\right)\right\}}{\sum\left\{L\left(X \cap T_{1}\right)\right\}}=\frac{4 \sum\left\{B\left(Y \cap T_{2}\right)\right\}}{\pi \sum\left\{A\left(X \cap T_{2}\right)\right\}}
$$

or:

$$
C P A_{V}=\frac{2 \sum I_{C I}}{\sum L_{C}}=\frac{4 \sum L_{C I}}{\pi \sum A_{C}}
$$

In the formula, $L_{C I}$ is the length of curve intercepts of $Y$ phase. By taking formula (13), (15) and (16) into formula (12), we can get that:

$$
A_{s}=\frac{C P A_{V}}{S_{V}}=\frac{\frac{S(Y)}{V(X)}}{\frac{S(\delta X)}{V(X)}}=\frac{2 \frac{\sum\left\{I\left(Y \cap T_{1}\right)\right\}}{\sum\left\{L\left(X \cap T_{1}\right)\right\}}}{2 \frac{\sum\left\{I\left(X \cap T_{1}\right)\right\}}{\sum\left\{L\left(X \cap T_{1}\right)\right\}}}=\frac{\sum\left\{I\left(Y \cap T_{1}\right)\right\}}{\sum\left\{I\left(X \cap T_{1}\right)\right\}}=\frac{\frac{4 \sum\left\{B\left(Y \cap T_{2}\right)\right\}}{\pi \sum\left\{A\left(X \cap T_{2}\right)\right\}}}{\frac{4 \sum\left\{\left(\delta X \cap T_{2}\right)\right\}}{\pi \sum\left\{A\left(X \cap T_{2}\right)\right\}}}=\frac{\sum\left\{B\left(Y \cap T_{2}\right)\right\}}{\sum\left\{B\left(\delta X \cap T_{2}\right)\right\}}
$$

The above formula can be rewritten as:

$$
\begin{gathered}
A_{S}=\frac{2 \sum I_{C I} / \sum L_{C}}{2 \sum I_{C} / \sum L_{C}}=\frac{\sum L_{C I}}{\sum L_{C}} \\
A_{S}=\frac{4 \sum L_{C I} /\left(\pi \sum A_{C}\right)}{4 \sum B_{C} /\left(\pi \sum A_{C}\right)}=\frac{\sum L_{C I}}{\sum B_{C}}
\end{gathered}
$$

It is clear that curve profile area density in surface is equal to the value that the intersections of curve intercepts of subset $Y$ intersecting with test line divided by the intersections of containing curve intersecting with test line, and also equal to the value that the length of curve intercepts of subset $Y$ divided by the length of containing boundary (or containing curve line) in 2D plane. So the key formula for $A_{S}$ is that:

$$
A_{S}=I_{I S}=C I D
$$

i.e.: curve profile area density in surface is equal to the curve intercept intersection density relied on intersection of containing curve (or curve profile 
intersection density in surface), and equal to the curve intercept density.

\section{EXAMPLE FOR TESTING}

Fig. 2 is an example for calculating curve profile area density in surface. Now let us use the square lattice test system to test $A_{S}$ of the particles in the sphere surface on $2 \mathrm{D}$ plane. First, counting $I_{C}$, the intersections of sphere surface (or the boundary of circle in 2D) intersecting with test line in horizontal and longitudinal directions. It is 56; Secondary, counting $I_{C l}$, the intersections of curve profiles (which show as curve intercepts in 2D, and are formed by the shadow particles intersecting with sphere surface), intersecting with test line in horizontal and longitudinal directions, it is 34 ; Finally calculating $A_{S}$ by formula (18), i.e.: $A_{S}=34 / 56=60.7 \%$. If we want to use formula (19) to calculating $A_{S}$ of the shadow particles in the sphere surface, the entire length of containing curve (or the perimeter of the circle in 2D plane) should be measured or calculated by the diameter of the circle (or by image analysis system) first, it is $18.21 \mathrm{~cm}$. Then, measure the lengths of curve intercepts of the shadow particles intersecting with the circle boundary one by one and sum the lengths, it is $11.28 \mathrm{~cm}$ in total. Finally calculating $A_{S}$ by formula (19), i.e.: $A_{S}=11.28 / 18.21=61.8 \%$. The results are close each other by aforesaid two methods. If we increase the sample size, the practical testing results will tend to be same by these two kinds of methods.

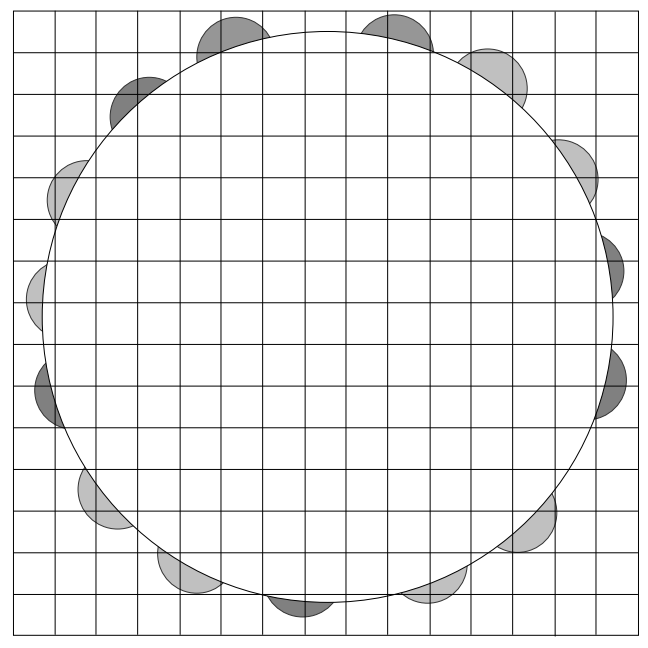

Fig. 2. An example for test of curve profiles area density in surface by square lattice test system.

\section{DISCUSSION}

1. About the correction for tissue shrinkage. Let the shrinkage coefficient of the curve spatial surface 3D tissue $(X)$ be $f_{\mathrm{S}, \mathrm{S}}$, the shrinkage coefficient of subset $Y$, which are formed by a 3D structure $Z$ intersecting with $X$, be $f_{\mathrm{S}, C P A}, A_{S}$ ' and $A_{S}$ be the curve profile area density in surface before and after tissue shrinkage correction. By the general correcting method for tissue shrinkage (Shen 1997, Shen and Shen 1991), we can get that:

$A_{S}=\frac{S(Y) \hbar f_{S, C P A}{ }^{-2}}{S(X) \hbar f_{S, S}{ }^{-2}}=\frac{C P A \hbar f_{S, C P A}{ }^{-2}}{S_{C} \hbar f_{S, S}{ }^{-2}}=A_{S \cdot} \hbar \frac{f_{S, S}{ }^{2}}{f_{S, C P A}{ }^{2}}$

If the coefficients of the two intersecting tissues are equal to each other, i.e.: $f_{\mathrm{S}, \mathrm{S}}=f_{S, C P A}$, then:

$$
A_{S}=A_{S}
$$

This means that it is not necessary to make correction to the parameter of $A_{S}$ for the tissue shrinkage if the shrinkage degree is same for these two intersecting tissues. In fact, as we know that curve profiles area is a part of containing surface, so we can reasonably suppose that: $f_{\mathrm{S}, \mathrm{S}}=f_{S, C P A}$, or $f_{\mathrm{S}, \mathrm{S}} \approx f_{S, C P A}$. Then we can get $A_{S}$ directly by formulas (18) or (19). The test method for the coefficient of tissue shrinkage is as same as that in Weibel's book (1979).

2. About the application for the parameter of $A_{S}$. $A_{S}$ can be used to quantitatively describe the amount of curve profile area of particles in unit surface area of containing membrane or membrane like structure. In biotissues, it can be used to quantitatively express the amount of mouth area in unit containing surface area, such as the mouth area of adenoduct of sweat gland and sebaceous gland in unit surface area of skin, of intestinal glands in unit surface area of intestine mucosa. It also can be used to quantitatively express the occupied area of some kind of cells on the side of basement membrane of gland in unit surface area of basement membrane. The area occupied by osteoblasts in unit surface area of bone trabecula, by tight junctions or gap junctions, intermediate junctions, desmosomes, microvilli, cilia and synapses etc. in unit surface area of cell membrane, by ribosomes in unit surface area of rough endoplasmic reticulum, by nuclear pores in unit surface area of nuclear membrane, by capillary pores in unit surface area of fenestrated capillary on the side of lumen, by alveolar pores or type II alveolar epithelium in unit surface area of 
pulmonary alveoli, or by synaptic vesicle in unit surface area of presynaptic membrane, etc. All above can be quantitatively described with the parameter of $A_{S}$.

3. About empty curve profile. By the definition of empty curve profile, any holes in membrane or membrane like structures could be considered as empty curve profiles. Glandular duct mouthes of all kinds of exocrine glands in surface of mucosa or skin, nuclear pores, alveolar pores and capillary pores etc. are just the examples of empty curve profiles. Empty curve profiles in 2D plane express as a structureless (hole or pore like structures). So we can't see any empty curve intersections actually, but an empty area, holes or pores or mouth, maybe with a boundary. The empty curve intercepts in 2D show as an interrupted or divided intercepts. In fact, it is also invisible. Therefore when a test line passing through this kind of empty area, interrupted or divided intercepts, it should be counted as an intersection with curve intercepts.

4. It is necessary to emphasize that the reference systems of curve profile area density in surface, curve intercept density, curve profile intersection density in surface (or curve intercept intersection density relied on intersections of containing curve), are separately the correspondent surface area of containing surface, the length of containing curve line, and the intersections of containing curve (or curve line) with test lines. The essential difference between the curve profile area density in surface and the curve profile area density depend just on the references system or containing space. The reference for the former is the containing surface, but that for the latter is the volume of containing space. The general used intersection density is different from the intersections density in containing intersection (or intersection density relied on intersection reference) just in containing system (or reference system) and physical unit. The intersection density, $I_{L}$, employ the length of test line in containing space in 2D for its reference system and its physical unit is numbers per $\mathrm{mm}$ or $\mu \mathrm{m}$ (Weibel, 1979, 1980; Collan, 1984; Shen and Shen, 1991; Howard and Reed, 1998). But the intersection density in containing intersection, $I_{I}$, employ the intersections in containing curve intersecting with test lines for its reference system with no physical unit.

\section{ACKNOWLEDGEMENT}

The study was partly supported by National Natural Science Foundation of China.

\section{REFERENCES}

Collan Y et al (1984). Stereology and morphometry in pathology. Kuopio: Kuopio University Press, 21-4.

Howard CV, Reed MG (1998). Unbiased stereology: three-dimensional measurement in microscopy. New York: Springer-Verlag New York Inc., BIOS Scientific Publishers, 107-18.

DeHoff RT (2000). Populations, samples, measurements and relations in stereology. Image Anal Stereol 19:1-8.

Shen H, Shen ZY (1991). Practical technique for biostereology. Guangzhou: Zhongshan University Publishing House, 80-86 (in Chinese).

Shen H (1997). Correcting methods of error for surface density, surface area and the ratio of surface area to volume due to tissue shrinkage: discussion with Weibel's approach. E J Pathol 3.4:974-06.

Weibel ER (1979). Stereological methods. Vol.1, Practical methods for biological morphometry. London: Academic Press, 31-122, 152-153

Weibel ER (1980). Stereological Methods Vol. 2, Theoretical Foundation. London, New York, Toronto, Sydney, San Francisco: Academic Press, 315. 